\title{
The Cambridge Symposium: September 2002
}

AJ Bron

\section{Eye (2003) 17, 851. doi:10.1038/sj.eye.6700618}

This Cambridge Symposium (Chairpersons: AJ Bron and D Coster) on the cornea was dedicated to one of the founders of ophthalmic science, Dr David Maurice, who died in the summer of 2002. His research interests encompassed ocular physiology and pharmacokinetics. During his research career, which spanned 50 years, he demonstrated a profound understanding of ocular pathology and as a consequence made many contributions to clinical practice.

Perhaps the greatest of his contributions was to identify the basis for corneal transparency. $\mathrm{He}$ recognised that to understand the effects of corneal oedema and scarring on vision, it was necessary to ask not why the cornea might become opaque, but why it was transparent in the first place. His 'lattice theory' proposed that the remarkable transparency of the cornea was due to the structural order of the stromal collagen fibrils, leading to a cancelling out of backscattered light by destructive interference. Over the years, this theory has been modified in detail but not in substance and is the basis of our current understanding.

He also designed instruments for the study of the living eye, which have been used for research in both animals and humans. Many of these still influence ophthalmic research and practice today. His development of the optical pachometer and specular comeal microscope has been critical to the evolution of modern cataract and corneal graft surgery. This gave us an understanding of the basis of corneal oedema and provided tools which have allowed us to assess endothelial viability pre- and postoperatively. It is now standard practice for eye banks to provide details of the endothelial cell density in donor corneas, measured by specular microscopy. His research too established the essential metabolic requirements of the corneal endothelium, which form the basis of modern, safe, irrigating solutions.

David Maurice also made major contributions to our understanding of ocular pharmacokinetics. He demonstrated the permeability characteristics of the ocular surface. He defined the paracellular pathways of the comeal epithelium and endothelium and focused our attention on the barriers to drug penetration and diffusion through the cornea and conjunctiva. His invention of the fluorometer permitted eye scientists to study aqueous and tear flow in near steady-state conditions. It has been used to measure aqueous flow in human subjects, to show its diurnal rhythm, autonomic control, and the effects of anti-glaucoma drugs. It has also allowed tear turnover to be measured and made it possible to define conditions such as dry eye and to demonstrate the contact time of topically instilled drugs.

I believe that many of the topics covered in this corneal symposium would have been of great interest to David Maurice. As is the custom at the Cambridge symposia, basic and clinical scientists of international standing provided us with an exciting overview of a wealth of subjects and their talks stimulated lively discussions between the speakers and the audience. We heard about corneal structure and the stem cells of the ocular surface, the regulation and role of the tears, the mechanisms and treatment of corneal infection, the latest techniques of corneal transplantation, and the factors determining graft tolerance. The meeting was an outstanding success and a fitting tribute to Dr Maurice.
Nuffield Laboratory of Ophthalmology Walton Street Oxford 0X2 6AW, UK

Correspondence:

AJ Bron

Tel: 01865224936

E-mail: anthony.bron@ eye.ox.ac.uk 